\title{
Characterization of a novel polyclonal anti-hypusine antibody
}

\author{
Yurika Nishiki ${ }^{1}$, Thomas B Farb², Jessica Friedrich², Krister Bokvist ${ }^{2}$, Raghavendra G Mirmira ${ }^{1,3^{*}}$ and Bernhard Maier ${ }^{1}$
}

\begin{abstract}
The translation factor elF5A is the only protein known to contain the amino acid hypusine, which is formed posttranslationally. Hypusinated elF5A is necessary for cellular proliferation and responses to extracellular stressors, and has been proposed as a target for pharmacologic therapy. Here, we provide the first comprehensive characterization of a novel polyclonal antibody (IU-88) that specifically recognizes the hypusinated elF5A. IU-88 will be useful for the investigation of elF5A biology and for the development of assays recognizing hypusinated elF5A.
\end{abstract}

Keywords: Hypusine; elF5A; Antibody; Recombinant proteins; Cell lines

\section{Introduction}

Eukaryotic translation initiation factor $5 \mathrm{~A}-1$ and $5 \mathrm{~A}-2$ (eIF5A-1 and eIF5A-2-collectively referred to here as eIF5A) are highly conserved proteins whose varied cellular functions include the binding of and nucleocytoplasmic shuttling of specific mRNAs (Kruse et al. 2000; Maier et al. 2010; Xu and Chen 2001), cellular proliferation (Nishimura et al. 2005), and posttranslational stress responses ( $\mathrm{Li}$ et al. 2010; Moore et al. 2008; Nishiki et al. 2013). Curiously, eIF5A is the only protein containing the amino acid hypusine, which is formed from a lysine residue in a posttranslational reaction involving the enzymes deoxyhypusine synthase (DHS) and deoxyhypusine hydroxylase (DHH) and the substrate spermidine (Park et al. 2010). In the complete absence of deoxyhypusine synthase mouse embryos die at a very early stage of development (Nishimura et al. 2012; Templin et al. 2011). Inhibition of hypusine formation has been suggested to confer cellular survival in certain stress states, such as infections, carcinogenesis, and obesity (Balabanov et al. 2007; Hauber et al. 2005; Robbins et al. 2010; Schwentke et al. 2012). Therefore, identification of the hypusinated form of eIF5A is paramount in the understanding of the biology of the protein. Nevertheless, identification of hypusinated eIF5A

\footnotetext{
* Correspondence: rmirmira@iu.edu

'Department of Pediatrics and the Herman B Wells Center for Pediatric Research, Indiana, University School of Medicine, Indianapolis, IN 46202, USA

${ }^{3}$ Departments of Medicine, Cellular and Integrative Physiology, and Biochemistry and Molecular Biology, Indiana University School of Medicine, Indianapolis, IN 46202, USA

Full list of author information is available at the end of the article
}

has remained a challenge, requiring tedious methods such as isoelectric focusing or two-dimensional gel electrophoresis of cellular extracts. Although prior studies reported the development of antibodies against hypusinated eIF5A (Bergeron et al. 1998; Cracchiolo et al. 2004), their characterizations were limited and utilities of these reagents were not described in subsequent reports. Here, we present the characterization of a novel anti-hypusine antibody reagent, IU-88. We demonstrate that IU-88 selectively recognizes either the deoxyhypusine or hypusine forms of eIF5A in vitro, and that IU-88 specifically recognizes the hypusinated form of eIF5A in cellular extracts by immunoblots and in whole cells by immunocytochemistry.

\section{Materials and methods}

\section{Cell culture, transfection and DHS inhibition}

Human 293T and rat INS-1(832/13) $\beta$ cells were cultured as described (Hohmeier et al. 2000). Cells were transiently transfected with plasmids encoding EGFPeIF5A, EGFP-eIF5A(K50A) and EGFP-DHS constructs using Lipofectamine 2000 (Invitrogen) for 16 hours before cell extraction or immunofluorescence analysis. The DHS inhibitor GC7 (Biosearch Technologies) was prepared and used in cell culture as previously described (Maier et al. 2010).

\section{Reactions in vitro}

For in vitro experiments, eIF5A protein was purified from E. coli as a GST fusion, after which the GST tag was proteolytically removed. DHS protein was purified

\section{实}


from E. coli as an N-terminal His6 fusion. Purified human $\mathrm{DHH}$ protein was purchased from OriGene. The hypusination reactions in vitro proceeded as previously published (Wolff et al. 2011).

\section{Antibodies and immunoblotting}

The rabbit polyclonal antibody IU-88 against hypusinated human eIF5A was generated in rabbits using the synthetic hypusine-containing peptide C-Ahx-STSKTG[hypusine] HGHAKV-amide by contract to $21^{\text {st }}$ Century Biochemicals. Monoclonal mouse pan-anti-eIF5A antibody was from BD Biosciences and anti-actin antibody was from MP Biomedicals. Immunoblot analysis was visualized using a LiCor Odyssey fluorescence system following electrophoresis on a 4-20\% SDS polyacrylamide gel (Maier et al. 2010). Primary antibodies were diluted 1:1500 (IU-88) and 1:10,000 (anti-pan-eIF5A).

\section{Fluorescence immunocytochemistry}

293T cells were fixed in 4\% paraformaldehyde and immunocytochemistry proceeded as previously described (Robbins et al. 2010). Antibody dilutions were 1:150 for IU-88 and 1:1000 for anti-pan-eIF5A. 4'6-diamidino-2phenylindole (DAPI) staining was used to visualize nuclei. A Zeiss LSM-710 microscope was used to visualize cells at magnification $\mathrm{x} 100$.
A

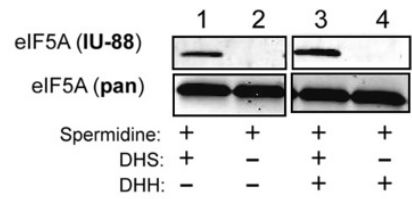

C

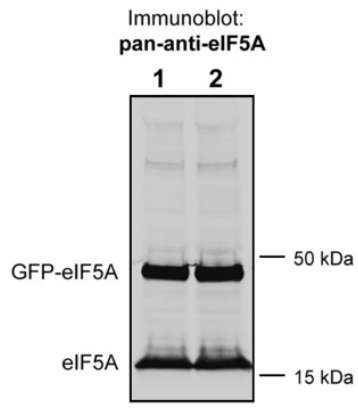

E

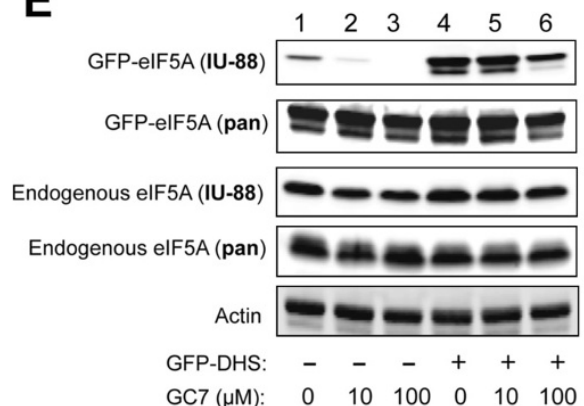

B

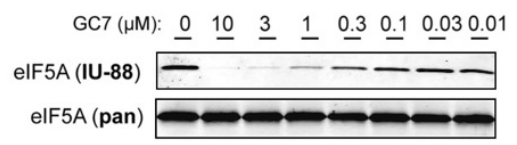

D

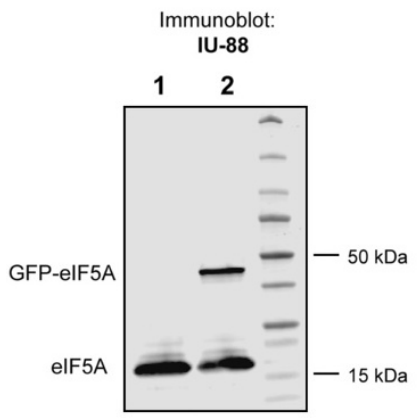

$\mathbf{F}$

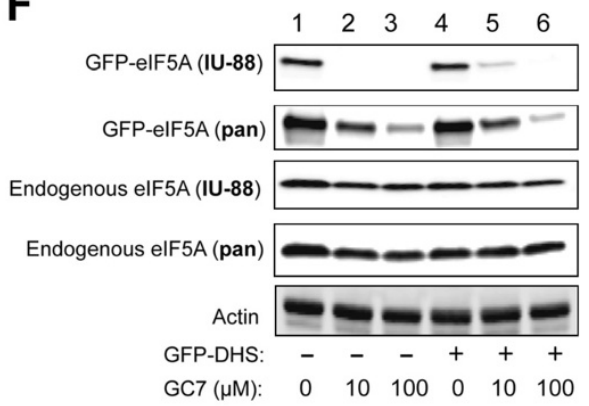

Figure 1 Immunoblot characterization of polyclonal antibody IU-88. $\boldsymbol{A}$, Recombinant human elF5A was treated in vitro with spermidine, DHS, and DHH as indicated, then subjected to polyacrylamide gel electrophoresis and immunoblots analysis using antibody IU-88 or a pan-antielF5A antibody (a BD monoclonal antibody); $\boldsymbol{B}$, Recombinant human elF5A was treated in vitro with spermidine, DHS, and different concentrations of GC7 as indicated, then subjected to polyacrylamide gel electrophoresis and immunoblot analysis using antibody IU-88 or a pan-anti-elF5A antibody; C, INS-1 $\beta$ cells were transfected with a plasmid encoding either GFP-elF5A(K50A) (Iane 1) or GFP-elF5A (lane 2), then cell extracts were subjected to polyacrylamide gel electrophoresis and immunoblots analysis using a pan-anti-elF5A antibody; $\boldsymbol{D}$, INS-1 $\beta$ cells were transfected with a plasmid encoding either GFP-elF5A(K50A) (lane 1) or GFP-elF5A (lane 2), then cell extracts were subjected to polyacrylamide gel electrophoresis and immunoblots analysis using antibody IU-88; $\boldsymbol{E}$, 293T cells were transfected with a plasmid encoding GFP-elF5A, with or without another plasmid encoding DHS (as indicated), and treated with different concentrations of GC7 as indicated. Cell extracts were then subjected to polyacrylamide gel electrophoresis and subsequent immunoblots analysis using antibody IU-88, a pan-anti-elF5A antibody, and an anti-actin antibody; $\boldsymbol{F}$, INS-1 cells were transfected with a plasmid encoding GFP-elF5A, with or without another plasmid encoding DHS (as indicated), and treated with different concentrations of GC7 as indicated. Cell extracts were then subjected to polyacrylamide gel electrophoresis and subsequent immunoblots analysis using antibody IU-88, a pan-anti-elF5A antibody, and an anti-actin antibody. 


\section{Results and discussion}

To determine if IU-88 specifically recognizes the deoxyhypusine or hypusine forms of eIF5A, we performed immunoblots of reactions in which recombinant human eIF5A was incubated in vitro with DHS, DHH, spermidine, and/or the potent DHS inhibitor GC7. Figure 1A shows that IU-88 is incapable of recognizing eIF5A when it is incubated with spermidine alone (lane 2) or with $\mathrm{DHH}+$ spermidine (lane 4). However, IU-88 recognized eIF5A when co-incubated with DHS+spermidine (lane 1) or DHS+DHH+spermidine (lane 3), suggesting that both the deoxyhypusine and hypusine forms of eIF5A are recognized (although it cannot be determined if both forms are recognized with equal affinity). Increasing the DHS concentration and time of incubation led to increasing eIF5A signal intensity in these studies (data not shown). Co-incubation of the reaction with DHS+spermidine with increasing concentrations of the DHS inhibitor GC7 (up to $10 \mu \mathrm{M}$ ) caused near-complete inhibition of eIF5A signal intensity (Figure 1B). Notably, the differences in eIF5A intensity in these studies were not because of differences in protein loading, since a pan-anti-eIF5A monoclonal antibody (BD) demonstrated equal loading (Figure $1 \mathrm{~A}$ and $\mathrm{B}$ ).

Next, we tested the ability of IU-88 to specifically recognize hypusinated eIF5A in whole cellular extract by immunoblotting. As shown in the full gel image in Figure $1 C$, when extracts from rat-derived INS-1 islet $\beta$ cells are used in immunoblotting, IU-88 recognizes only a single protein species at $\sim 17 \mathrm{kDa}$, corresponding to

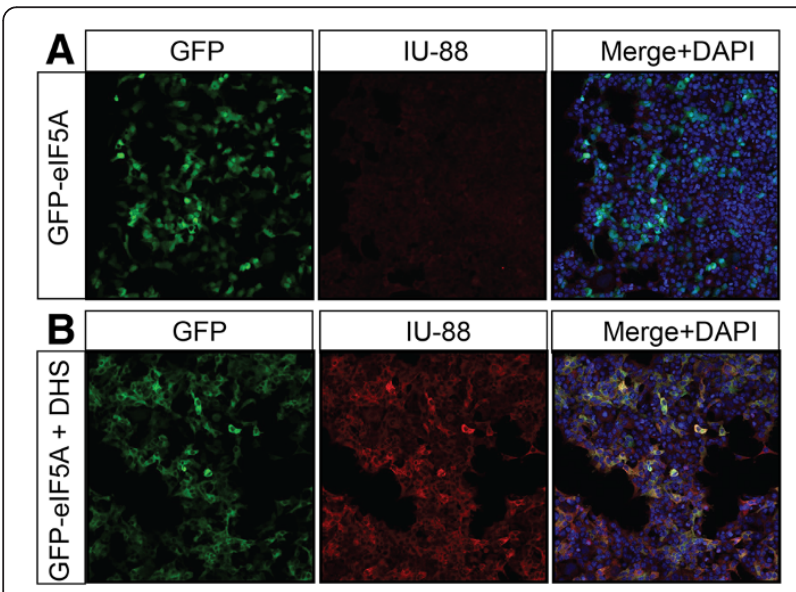

Figure 2 Immunocytochemistry of 293T cells using antibody IU-88. $\boldsymbol{A}, 293$ T cells were transfected with a plasmid encoding GFP-elF5A, then immunostained using IU-88 and counterstained with DAPI to visualize nuclei; $\boldsymbol{B}$, 293T cells were transfected with plasmids encoding GFP-elF5A and DHS, then immunostained using IU-88 and counterstained with DAPI to visualize nuclei. In panels $\boldsymbol{A}$ and $\boldsymbol{B}$, GFP is visualized in the green channel, IU-88 is visualized in the red channel, and DAPI is visualized in the blue channel. Magnification $\times 100$ the known molecular weight of eIF5A. Transfection of a plasmid encoding either a human EGFP-eIF5A(K50A) fusion protein (which is not capable of being hypusinated) or a human EGFP-eIF5A fusion protein results in the appearance of a protein species at $\sim 44 \mathrm{kDa}$ only with the EGFP-eIF5A transfection (Figure 1C, compare lanes 1 and 2). When these transfections are immunoblotted using an antibody against GFP, both EGFP-eIF5A (K50A) and EGFP-eIF5A are recognized (Figure 1D). These data demonstrate specificity of IU-88 in recognizing only eIF5A in total cellular protein, and also suggest that IU-88 only recognizes transfected eIF5A proteins that have the capability to be hypusinated. To investigate in greater detail the utility of IU-88 to distinguish hypusination in cellular extracts, we performed additional studies in human-derived 293T cells and rat-derived INS-1 cells. As shown in Figure 1E, when human 293T cells are transfected with GFP-eIF5A, a weak but detectable signal corresponding to GFP-eIF5A is observed using IU-88 (lane 1). This signal decreases further upon co-incubation with increasing concentrations of GC7 (Figure 1E, lanes 2 and 3), suggesting that IU-88 is recognizing the hypusine-specific form. Interestingly, when exogenous DHS is introduced by co-transfection of a GFP-DHS fusion protein-encoding vector, there is a dramatic increase in GFP-eIF5A signal as detected by IU-88 (Figure 1E, lane 4) with corresponding decrease in the presence of GC7 (lanes 5 and 6), suggesting that DHS protein levels may be limiting in the ability of $293 \mathrm{~T}$ cells to hypusinate eIF5A - a finding that is also observed in human-derived HeLa cells (Lee et al. 2009). INS $-1 \beta$ cells, by contrast, reveal a significantly different picture. As shown in Figure 1F, transfection of a plasmid encoding GFP-DHS did not enhance the signal observed with either GFP-eIF5A or endogenous eIF5A, suggesting that DHS is not limiting in the ability of INS-1 cells to hypusinate eIF5A. Interestingly, whereas increasing GC7 concentrations reduce the GFP-eIF5A signal observed with IU-88, it also reduces the signal observed with the pan-anti-eIF5A antibody (Figure 1F). This result suggests that INS-1 $\beta$ cells may be unique in their requirement for hypusination to maintain production of eIF5A itself.

Figure $1 \mathrm{E}$ and $\mathrm{F}$ show that $\mathrm{GC7}$ incubation also reduces, but only slightly, the level of endogenous eIF5A, as detected by IU-88 (and perhaps more-so in INS-1 cells than in 293T cells). Because IU-88 measures only steady-state levels of hypusinated eIF5A (as opposed to rate of hypusine formation-as measured by $3 \mathrm{H}$-spermidine uptake studies, ref. (Maier et al. 2010)), this observation may reflect the long half-life (6-24 h) of the hypusinated eIF5A protein in mammalian cells (Gerner et al. 1986; Maier et al. 2010). More effective depletion of the hypusinated eIF5A species with GC7 may require longer periods of incubation. 
Because IU-88 shows specificity for hypusinated eIF5A, we next asked whether IU-88 can recognize protein in the context of fluorescence immunocytochemistry. 293T cells were transfected with a plasmid encoding GFP-eIF5A, then stained with DAPI (to visualize nuclei) and immunostained using IU-88. As shown in Figure 2A staining intensity with IU-88 was weak, consistent with the immunoblot in Figure 1D. However, when cells were cotransfected with GFP-DHS, a striking increase in cytoplasmic staining was observed with IU-88 (Figure 2B). A notable observation in Figure 2 is the apparent relocalization of eIF5A from a pan-nuclear/cytoplasmic distribution to a primarily cytoplasmic distribution in the presence of DHS overexpression (c.f. GFP-eIF5A staining in Figure 2A and B). This result suggests that hypusinated eIF5A may occupy primarily a cytoplasmic distribution, as proposed in prior studies (Lee et al. 2009; Maier et al. 2010). However, recent studies have also implicated a role for acetylation in eIF5A compartmentation (Ishfaq et al. 2012), suggesting perhaps a more complex interplay between hypusination and other modifications in the function and subcellular localization of the factor.

Taken together, our results verify the specificity and utility of IU-88 in detecting a specifically modified form of eIF5A. Depending upon the application, an important caveat to the use of IU-88 is its inability to distinguish between the deoxyhypusinated and hypusinated forms of eIF5A. Although the relative significance of the deoxyhypusinated vs. hypusinated forms of eIF5A remains unclear, the low substrate $\mathrm{Km}$ of $\mathrm{DHH}$ relative to DHS means that the majority of eIF5A in cells is likely present in the fully hypusinated form (Park et al. 2010). Nevertheless, IU-88 represents an especially useful reagent for the assessment of at least the activity of DHS in cells. Also, because most pharmacologic approaches to inhibiting the hypusination reaction have focused on inhibition of the higher Km enzyme DHS, IU-88 would also serve as an important reagent for assessing DHS activity in drug screening studies.

\section{Availability and requirements}

None.

\section{Competing interests}

The authors declare that they have no conflicts of interest relative to the work presented in this article.

\section{Authors' contributions}

YN, TBF, JF, KB, RGM, and BM performed research. RGM wrote the paper. All authors read and approved the final manuscript.

\section{Acknowledgements}

This work was supported by grants R01 DK060581 (to RGM) and T32 DK064466 (to YN) from the National Institutes of Health and by a grant from the Juvenile Diabetes Research Foundation (to RGM).

\section{Author details}

'Department of Pediatrics and the Herman B Wells Center for Pediatric Research, Indiana, University School of Medicine, Indianapolis, IN 46202, USA. ${ }^{2}$ Lilly Research Labs, Eli Lilly \& Co, Indianapolis, IN 46285, USA. ${ }^{3}$ Departments of Medicine, Cellular and Integrative Physiology, and Biochemistry and Molecular Biology, Indiana University School of Medicine, Indianapolis, IN 46202, USA.

Received: 1 July 2013 Accepted: 26 August 2013

Published: 29 August 2013

\section{References}

Balabanov S, Gontarewicz A, Ziegler P et al (2007) Hypusination of eukaryotic initiation factor $5 \mathrm{~A}$ (elF5A): a novel therapeutic target in BCR-ABL-positive leukemias identified by a proteomics approach. Blood 109:1701-1711. doi:10.1 182/blood-2005-03-037648

Bergeron RJ, Weimar WR, Müller R et al (1998) Synthesis of reagents for the construction of hypusine and deoxyhypusine peptides and their application as peptidic antigens. J Med Chem 41:3888-3900. doi:10.1021/jm980389p

Cracchiolo BM, Heller DS, Clement PM et al (2004) Eukaryotic initiation factor 5A1 (elF5A-1) as a diagnostic marker for aberrant proliferation in intraepithelial neoplasia of the vulva. Gynecol Oncol 94:217-222. doi:10.1016/j.ygyno.2004. 03.018

Gerner EW, Mamont PS, Bernhardt A, Siat M (1986) Post-translational modification of the protein-synthesis initiation factor elF-4D by spermidine in rat hepatoma cells. Biochem J 239:379-386

Hauber I, Bevec D, Heukeshoven J et al (2005) Identification of cellular deoxyhypusine synthase as a novel target for antiretroviral therapy. J Clin Invest 115:76-85. doi:10.1172/JCl200521949

Hohmeier HE, Mulder H, Chen G et al (2000) Isolation of INS-1-derived cell lines with robust ATP-sensitive $\mathrm{K}+$ channel-dependent and -independent glucosestimulated insulin secretion. Diabetes 49:424-430

Ishfaq M, Maeta K, Maeda S et al (2012) Acetylation regulates subcellular localization of eukaryotic translation initiation factor $5 \mathrm{~A}$ (elF5A). Febs Lett 586:3236-3241. doi:10.1016/j.febslet.2012.06.042

Kruse M, Rosorius O, Kratzer F et al (2000) Inhibition of CD83 cell surface expression during dendritic cell maturation by interference with nuclear export of CD83 mRNA. J Exp Med 191:1581-1590

Lee SB, Park JH, Kaevel J et al (2009) The effect of hypusine modification on the intracellular localization of elF5A. Biochem Biophys Res Commun 383:497-502. doi:10.1016/j.bbrc.2009.04.049

$\mathrm{Li} \mathrm{CH}$, Ohn T, Ivanov P et al (2010) elF5A promotes translation elongation, polysome disassembly and stress granule assembly. PLOS ONE 5:e9942. doi:10.1371/journal.pone.0009942

Maier B, Ogihara T, Trace AP et al (2010) The unique hypusine modification of elF5A promotes islet beta cell inflammation and dysfunction in mice. J Clin Invest 120:2156-2170. doi:10.1172/JC138924

Moore CC, Martin EN, Lee G et al (2008) Eukaryotic Translation Initiation Factor 5A Small Interference RNA-Liposome Complexes Reduce Inflammation and Increase Survival in Murine Models of Severe Sepsis and Acute Lung Injury. J Infect Dis 198:1407-1414. doi:10.1086/592222

Nishiki Y, Adewola A, Hatanaka M et al (2013) Translational Control of Inducible Nitric Oxide Synthase by p38 MAPK in Islet $\beta$-Cells. Mol Endocrinol 27:336-349. doi:10.1210/me.2012-1230

Nishimura K, Murozumi K, Shirahata A et al (2005) Independent roles of elF5A and polyamines in cell proliferation. Biochem J 385:779-785. doi:10.1042/ BJ20041477

Nishimura K, Lee SB, Park JH, Park MH (2012) Essential role of elF5A-1 and deoxyhypusine synthase in mouse embryonic development. Amino Acids 42:703-710. doi:10.1007/s00726-011-0986-z

Park MH, Nishimura K, Zanelli CF, Valentini SR (2010) Functional significance of elF5A and its hypusine modification in eukaryotes. Amino Acids 38:491-500. doi:10.1007/s00726-009-0408-7

Robbins RD, Tersey SA, Ogihara T et al (2010) Inhibition of deoxyhypusine synthase enhances islet beta cell function and survival in the setting of endoplasmic reticulum stress and type 2 diabetes. J Biol Chem 285:39943-39952. doi:10.1074/jbc.M110.170142

Schwentke A, Krepstakies M, Mueller A-K et al (2012) In vitro and in vivo silencing of plasmodial dhs and elf-5a genes in a putative, non-canonical RNAi-related pathway. BMC Microbiol 12:1-1. doi:10.1186/1471-2180-12-107 
Templin AT, Maier B, Nishiki Y et al (2011) Deoxyhypusine synthase haploinsufficiency attenuates acute cytokine signaling. Cell Cycle 10:1-7. doi:10.4161/cc.10.7.15206

Wolff EC, Lee SB, Park MH (2011) Assay of deoxyhypusine synthase activity. Methods Mol Biol 720:195-205. doi:10.1007/978-1-61779-034-8_12

$\mathrm{Xu} \mathrm{A}$, Chen KY (2001) Hypusine is required for a sequence-specific interaction of eukaryotic initiation factor $5 \mathrm{~A}$ with postsystematic evolution of ligands by exponential enrichment RNA. J Biol Chem 276:2555-2561. doi:10.1074/jbc. M008982200

doi:10.1186/2193-1801-2-421

Cite this article as: Nishiki et al:: Characterization of a novel polyclonal anti-hypusine antibody. SpringerPlus 2013 2:421.

Submit your manuscript to a SpringerOpen ${ }^{\circ}$ journal and benefit from:

- Convenient online submission

- Rigorous peer review

- Immediate publication on acceptance

- Open access: articles freely available online

- High visibility within the field

- Retaining the copyright to your article

Submit your next manuscript at $\gg$ springeropen.com 